BMJ Surgery, Interventions, $\&$ Health Technologies

\section{Tissue stress from laparoscopic grasper use and bowel injury in humans: establishing intraoperative force boundaries}

To cite: Khan AF,

MacDonald MK, Streutker C, et al. Tissue stress from laparoscopic grasper use and bowel injury in humans: establishing intraoperative force boundaries. BMJ Surg Interv Health Technologies 2021;3:e000084. doi:10.1136/ bmjsit-2021-000084

AFK and MKM are joint first authors.

Received 01 March 2021 Accepted 01 June 2021

Check for updates

(c) Author(s) (or their employer(s)) 2021. Re-use permitted under CC BY-NC. No commercial re-use. See rights and permissions. Published by BMJ.

${ }^{1}$ CIGITI Lab, University of Toronto, Toronto, Ontario, Canada

${ }^{2}$ Laboratory Medicine and Pathobiology, St Michael's Hospital, Toronto, Ontario, Canada

${ }^{3}$ General Surgery, St Michael's Hospital, Toronto, Ontario, Canada

Correspondence to Dr Amanda Farah Khan; amanda.khan@ucalgary.ca

\section{ABSTRACT}

Objectives We aim to determine what threshold of compressive stress small bowel and colon tissues display evidence of significant tissue trauma during laparoscopic surgery.

Design This study included 10 small bowel and 10 colon samples from patients undergoing routine gastrointestinal surgery. Each sample was compressed with pressures ranging from $100 \mathrm{kPa}$ to $600 \mathrm{kPa}$. Two pathologists who were blinded to all study conditions, performed a histological analysis of the tissues. Experimentation: November 2018-February 2019. Analysis: March 2019May 2020.

Setting An inner-city trauma and ambulatory hospital with a 40-bed inpatient general surgery unit with a diverse patient population.

Participants Patients were eligible if their surgery procured healthy tissue margins for experimentation (a convenience sample). 26 patient samples were procured; 6 samples were unusable. 10 colon and 10 small bowel samples were tested for a total of 120 experimental cases. No patients withdrew their consent.

Interventions A novel device was created to induce compressive "grasps" to simulate those of a laparoscopic grasper. Experimentation was performed ex-vivo, in-vitro. Grasp conditions of $0-600 \mathrm{kPa}$ for a duration of $10 \mathrm{~s}$ were used.

Results Small bowel (10), M:F was 7:3, average age was 54.3 years. Colon (10), M:F was 1:1, average age was 65.2 years. All 20 patients experienced a significant difference $(p<0.05)$ in serosal thickness post-compression at both 500 and $600 \mathrm{kPa}$ for both tissue types. A logistic regression analysis with a sensitivity of $100 \%$ and a specificity of $84.6 \%$ on a test set of data predicts a safety threshold of $329-330 \mathrm{kPa}$.

Conclusions A threshold was discovered that corresponded to both significant serosal thickness change and a positive histological trauma score rating. This "force limit" could be used in novel sensorized laparoscopic tools to avoid intraoperative tissue injury.

\section{INTRODUCTION}

Laparoscopic graspers are used extensively in minimally invasive surgery, primarily to lift and mobilize delicate anatomical tissues
Significance of this study

What is already known about this subject?

- To the authors' knowledge, this will be the first study to investigate the upper limit of force by laparoscopic graspers in human tissues with a histological analysis of cellular damage. Previous studies used either a porcine model or used human tissues without a comprehensive histological analysis.

What are the new findings?

- Using our logistic regression analysis, our data points in the direction of establishing a maximum force cut-off starting at $329 \mathrm{kPa}$ on average for human gastrointestinal tissues using a $50 \%$ threshold, however very large safety margins should be considered and used. The tissue trauma score regression model was able to achieve a sensitivity of $76.9 \%$ and a specificity of $100 \%$ on a test set of data of $20 \%$ of cases.

\section{How might these results affect future} research or surgical practice?

- Our paper adds strong evidence for the establishment of an upper limit of atraumatic force in gastrointestinal human tissue and argues that this force cut-off should be used intraoperatively via laparoscopic grasper "smart" tools. The two logistic regression equations presented in this manuscript could be used in a tool intraoperatively, provided that the features used could be measured in real-time.

for better visualization and access. ${ }^{1}$ However, serious iatrogenic complications from the improper use of laparoscopic graspers in bowel surgery can occur and include: bowel perforation, serosal tears and postoperative adhesion formation. ${ }^{2}$ Bowel perforation is an especially severe complication because it is associated with a high morbidity and mortality rate (as high as $3.6 \%$ ). ${ }^{3}{ }^{4}$ Tang et $a l$ studied the removal of the gallbladder, a routine gastrointestinal laparoscopic procedure. When analyzing consequential and 
inconsequential errors committed using various surgical instruments, the error probability of holding graspers and dissecting graspers were higher than those of other surgical tools such as the electrosurgical hook knife. They found that perforation of the gallbladder and bleeding from liver injury were mainly caused by the use of excessive force or dissection in the wrong tissue planes. ${ }^{5}$

Careful evaluation of these statistics is especially pertinent in light of the fact that 100 patients a day die from iatrogenic injuries in US hospitals, with $40 \%$ of these injuries occurring in the operating room. ${ }^{6}$ In Canada, Baker $e t a l$, studied adverse events occurring in hospitals across five different provinces. They found that $7.5 \%$ of all patients admitted to acute care hospitals experienced one or more adverse event(s), with $51.4 \%$ of all adverse events arising from surgery. They judged that $36.9 \%$ of these adverse events were highly preventable. ${ }^{8}$

Unfortunately, there has been a dearth of studies that attempt to quantify the interaction of the grasper-tissue interface at a histological level to quantify which load forces tissue injuries occur at. This topic is particularly important to explore, because researchers have found that the handle and tip forces in laparoscopic graspers differ significantly from conventional graspers used in "open-approach" surgeries, which can lead to inappropriate force magnitudes and tissue damage..$^{9} 10$

\section{Complex mechanical response of tissue to compression}

It is challenging to accurately quantify and model biological soft tissues' multifaceted and complex behavior in response to the compressive force exerted by laparoscopic graspers. The mechanical response of tissue is based on two factors: (a) the inherent mechanical properties of that tissue and (b) the environmental loading characteristics it is subjected to. The small and large bowel are composed of multiple tissue layers. Within each layer, different fibers are distributed according to specific spatial orientations, which creates a strongly anisotropic configuration where measured properties varies along its different axes. ${ }^{11}$

\section{Grasper jaw geometry and stress on tissues}

Laparoscopic graspers have jaws traditionally made from stainless steel due to its durability and ease of sterilization. The main disadvantage of using metal is that metal is a much stiffer material than delicate gastrointestinal tissues and as such, compressing tissue with metal graspers can cause damage at the cellular level (such as mechanical destruction of the cell membrane or nucleus) or tissue level (such as rupture of muscle fibers or ischemia from the destruction of blood vessels). ${ }^{12}$ Graspers also come in a variety of jaw geometries and teeth profiles such as straight or flared, fenestrated with waves or solid and single or dual action, which contribute both to their function and damage potential. For example, when a grasper's jaw is serrated, increasing the size of the teeth will help prevent slippage but also causes more damage to tissue. ${ }^{13}$ Cheng and Hannaford investigated this relationship and created both a two-dimensional and three-dimensional finite element analysis study of calculated von Mises stress distributions under compression loads in a grasper and liver tissue model. ${ }^{14}$ They found that in the twodimensional plane strain model, that $80 \%$ of the stress in the area directly beneath the grasper was over $300 \mathrm{kPa}$, which is over the damage limit they elucidated in their previous work of $240 \mathrm{kPa}$ for liver tissue. ${ }^{15}$

\section{Is porcine tissue an accurate surrogate for human tissues?}

Previous studies exploring grasper jaw and tissue interactions have mostly centered on porcine tissue studies. This is due to the vast logistical and ethical challenges involved in human tissue experimentation and the previous assumption that porcine tissues are close enough to human tissues to be a surrogate model. Christensen $e t$ als work with porcine and human bowel tissues casts doubt on this assumption; however, as they found that human tissues were stronger, stiffer and less compliant than porcine tissue. Porcine tissue was able to stretch almost twice as much as human bowel tissue (with an elastic modulus of $1.83 \mathrm{MPa}$ and 5.18 , respectively), while human bowel tissue had a higher ultimate average strength $(0.58 \mathrm{MPa}$ compared with 0.87 for human tissues). ${ }^{16}$ Heijnsdijk et al also found that the inter-individual variability in perforation forces is quite large and that bowel strength could differ by a factor of two between patients. ${ }^{17}$

\section{Establishing safe tissue force boundaries in humans with a histopathological analysis}

This study aims to investigate the relationship between grasper jaw forces and human small and large bowel (colon) tissues. These tissue types were specifically chosen for two reasons: first, they are the most clinically relevant in relation to repeated grasp injury and second, bowel is one of the most delicate tissues in the human body. van der Voort $e t a l^{4}$ found that the overall incidence of laparoscopy-induced bowel injury was $0.36 \%$. The small intestine was most frequently injured $(55.8 \%)$, followed by the large intestine $(38.6 \%) .{ }^{3}$

To the authors' knowledge, this will be the first study to investigate the upper limit of force by laparoscopic graspers in human tissues with a histological analysis of cellular damage. A histological model was chosen to objectively and quantifiably understand how the intestinal tissue structure is microscopically affected as a result of mechanical loading. These data will be important as we move into an age of "smart surgical tools" that can quantify tool-tissue force interactions.

\section{MATERIALS AND METHODS}

\section{Study design and experimental protocol}

We previously created a prototype device, the Simple Crush Apparatus for Tissue to produce discrete grasp forces on human gastrointestinal tissue to test feasibility and workflow for human tissue experimentation. ${ }^{18}$ This current paper aims to build on the work of Chandler et 
A Experimental System

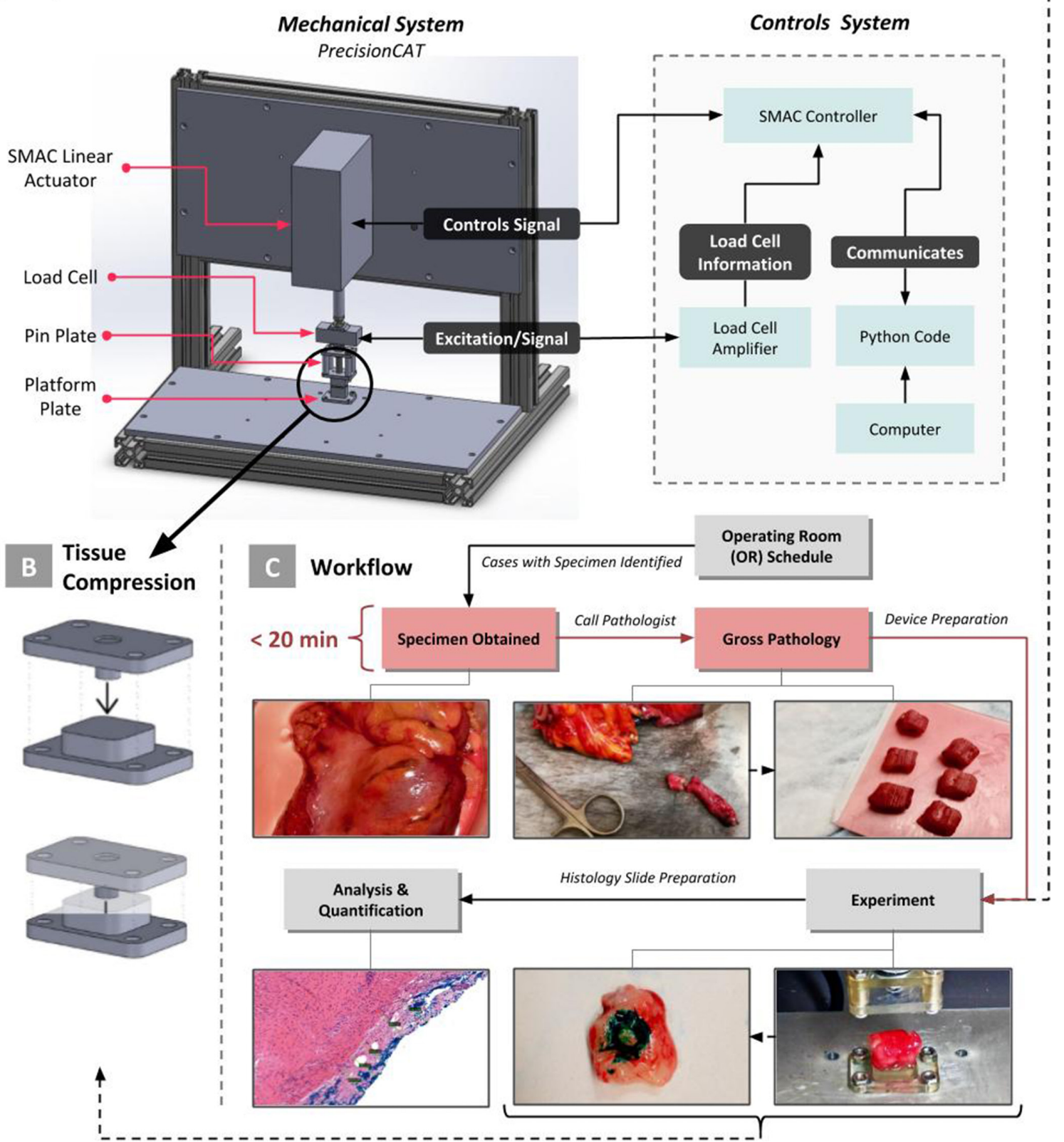

Figure 1 The Precision Crush Apparatus for Tissue (PrecisionCAT) crush apparatus and experimental workflow. (A) The experimental system consists of a precision linear actuator, load cell and indentation pin plates that is designed to simulate the compressive "grasps" of a laparoscopic grasper on tissue. (B) A close-up view of the indentation pin. The linear actuator brings the indentation pin plate into contact with the test platform plate. (C) The experimental workflow consists of identifying operations with appropriate tissue specimens, obtaining the removed specimen, calling the pathologist on-call who reviewed the gross specimen and sectioned out healthy tissue for experimentation, performing the full set of experiments within 20 min of tissue removal from the body and then creating histology slides for microscopic analysis.

$a l^{19}$ in defining an upper force limit for human small and large bowel tissue with a more sophisticated and accurate device than our previous work. To this end, a new custom device called the Precision Crush Apparatus for Tissue (PrecisionCAT) was created (figure 1A). Patients were consented by their operating surgeon, using a standard surgical consent form, which at our institution, includes a provision for using excess surgical tissues (not needed for surgical pathology) for research purposes. Patients were given an information form as well, that included a contact number if they chose to withdraw their sample from the study at any time postoperation. All methods were performed in accordance with the relevant guidelines and regulations of our institution and ethics office.

Each patient had six $1 \mathrm{~cm} \times 1 \mathrm{~cm}$ tissue samples cut from their usable tissue. Each sample was loaded onto the grasper plate of the PrecisionCAT serosa side up on a small cellulose-fiber sheet to avoid tissue slippage, and 


\section{A Layers of the Gastrointestinal Tract}

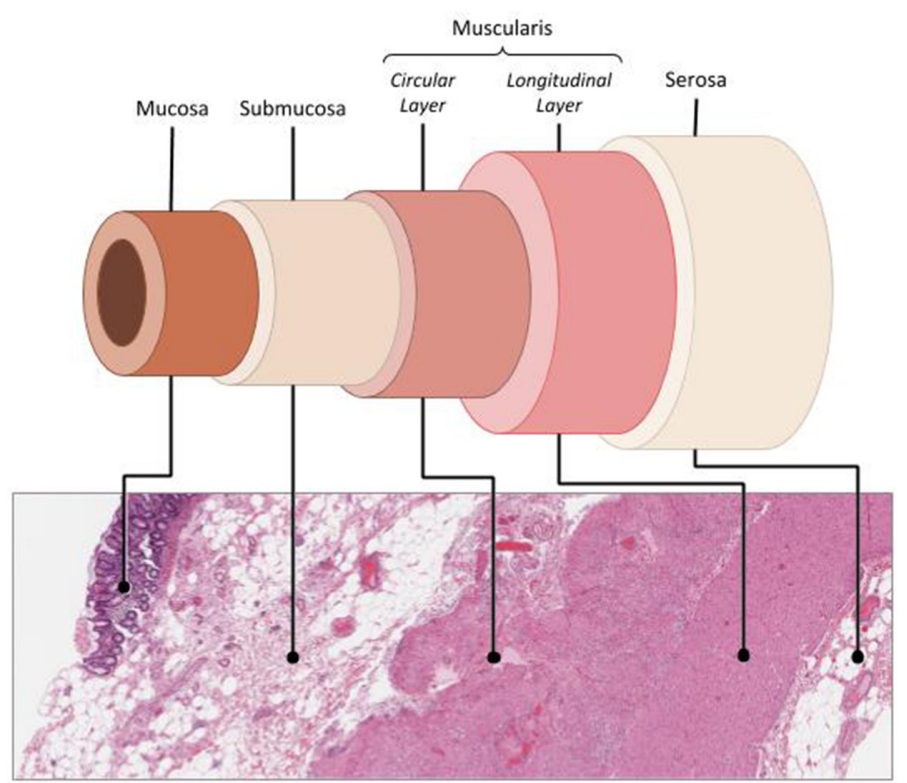

B Control vs. Compressed Tissue

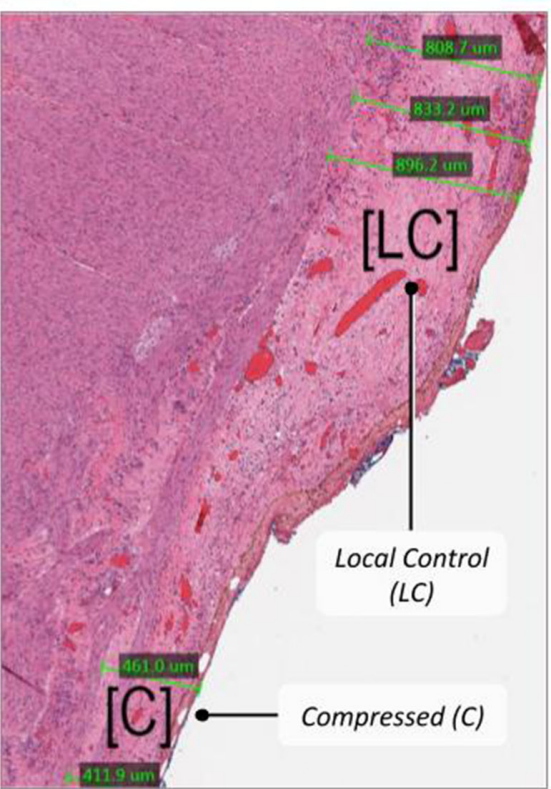

C Trauma Scores
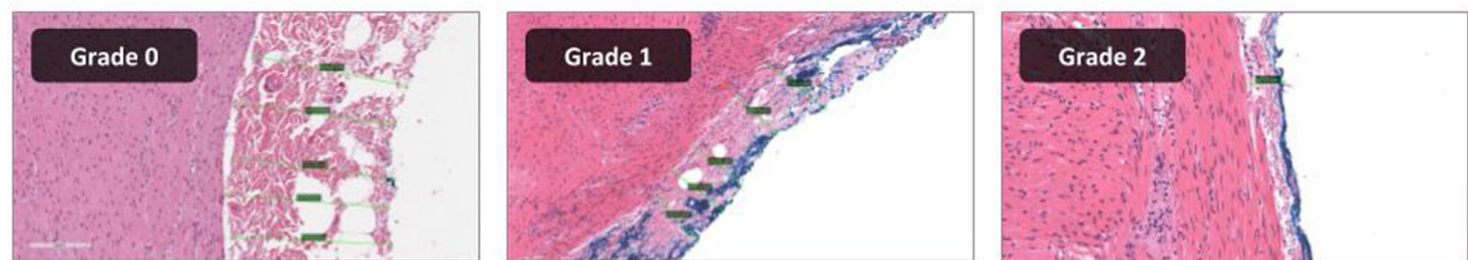

Figure 2 Gastrointestinal tract layers and Trauma Score. (A) The layers of the gastrointestinal tract, which comprise both small bowel and colon. (B) An example of tissue that has been experimentally compressed where $\mathrm{C}$ is the area of compression and LC a local control area. (C) Representative histological slides for each Trauma Score outlined in table 1. As there were no samples graded with a Trauma Score of 3 , a representative image is excluded.

one at a time, a discrete force was exerted on it ranging from 0 to $11.8 \mathrm{~N}(0-600 \mathrm{kPa})$. Specifically forces of $2 \mathrm{~N}$, $3.9 \mathrm{~N}, 5.9 \mathrm{~N}, 7.8 \mathrm{~N}, 9.8 \mathrm{~N}$ and $11.8 \mathrm{~N}$ were used, or using our precision pin plate with known surface area of 19.6 $\mathrm{mm}^{2}$, calculated pressures of $0 \mathrm{kPa}, 100 \mathrm{kPa}, 200 \mathrm{kPa}$, $300 \mathrm{kPa}, 400 \mathrm{kPa}, 500 \mathrm{kPa}$ and $600 \mathrm{kPa}$. Each simulated grasp was for a duration of $10 \mathrm{~s}$. This is consistent with our previous protocol which used precision weights and the same diameter of pin plate to generate equivalent force and pressure. These metrics were chosen to be consistent with the mean grasp force and $95 \%$ of grasp time elucidated by the Blue DRAGON system for laparoscopic tasks $(8.52 \mathrm{~N} \pm 2.77 \mathrm{~N}$ and $8.86 \mathrm{~s} \pm 7.06 \mathrm{~s})$, Zhou et al's tribology studies $(0-16 \mathrm{~N}$ with significant tissue damage achieved past $13 \mathrm{~N})$ and Chandler et al's protocol $(0-300 \mathrm{kPa}$ grasps with $10 \mathrm{~s}$ duration). ${ }^{420} 21$ The order in which tissues were compressed with each amount of force was randomized so that the pathologists did not know the tissue's loading condition. After each tissue sample was compressed, it was cut in half and processed for histology. On average, from the time the sample was removed from the patient to the end of the full experimental protocol, the research team took only $20 \mathrm{~min}$, to maximally preserve tissue integrity. When analyzing the slides, the two pathologists were again blinded to the amount of force each tissue was subjected to and slides were analyzed out of order to prevent bias.

\section{Experimental test equipment}

A test system (figure 1A, Build of Materials in online supplemental appendix) was developed based on Chandler $e t a l$ 's work, to apply mechanically controlled "grasps" to tissue samples that are characteristic of those sustained intraoperatively. Key requirements were similar to Chandler's: the test system should apply compressive grasps where the loading rate, peak stress and hold time are directly controlled and the grasper plate position precisely known. The ensuing system, named the PrecisionCAT, uses a linear actuator with $0.1 \mu \mathrm{m}$ resolution (LCA50025-72-1F-3, SMAC Moving Coil Actuators, California, USA) to drive together two "grasp plates" (representing the grasper jaws) and thus compress a sample of target tissue. The actuator was controlled via the SMAC LAC-1 servo motor controller (SMAC Moving Coil Actuators, California, USA) and used force data from the load cell to regulate velocity and position. The load cell used was 
Table 1 Tissue Trauma Scoring criteria

\begin{tabular}{ll}
\hline Trauma Score & Cellular architecture \\
\hline Grade 0 (no trauma to the serosa) & $\begin{array}{l}\text { Nuclei of serosal and bounding muscularis externa cells are smooth, oval and } \\
\text { uniformly shaped. }\end{array}$ \\
Grade 1 (minor trauma to the serosa) & $\begin{array}{l}\text { Elongation and mild hyperchromasia of nuclei in muscle/connective tissue in } \\
\text { both the serosa and muscularis propria externa outer longitudinal layer, but in } \\
\text { less than } 50 \% \text { of cells in our region of interest (ROI), representing trauma to the } \\
\text { cells. }\end{array}$ \\
Grade 2 (significant trauma to the serosa) & $\begin{array}{l}\text { Clear and significant damage to the serosa and muscularis propria externa outer } \\
\text { longitudinal layer, with more than } 50 \% \text { of nuclei in the cells in our ROI appearing } \\
\text { significantly elongated and thinned and there is the presence of multiple } \\
\text { hyperchromatic nuclei. }\end{array}$ \\
Grade 3 (complete denudation of the serosa) & $\begin{array}{l}\text { The serosa and muscularis externa longitudinal layers are both compressed, } \\
\text { with evidence of denudation of the serosa and trauma extending to the } \\
\text { muscularis propria inner circular layer. }\end{array}$
\end{tabular}

a high precision compression-link load cell (LCM-703-25, Omega Engineering, Connecticut, USA) coupled with a precision differential instrumentation amplifier (DMD465, Omega Engineering, Connecticut, USA) to amplify and convert the load cell's voltage to a larger voltage for digitization. Position and load data was synchronously recorded at $31 \mathrm{~Hz}$. The grasp plates were rapid prototyped at $75 \mu \mathrm{m}$ resolution using a Somos WaterShed XC 11122 (ZRapid SL600, Jiangsu, China). The plates' geometry is not based on a specific grasper jaw with fenestrations, but instead, comprise a smooth bottom test platform and the top, of a pin plate with an indentation tip, with the pin plate being driven closer to the test platform plate by the linear actuator (figure 1B). The cylindrical pin was fabricated with a known diameter of $5 \mathrm{~mm}$ and surface area of $19.6 \mathrm{~mm}^{2}$ to allow for pressure to be calculated from force for a standardized measurement.

The geometry of the indentation tip and its size relative to the test material and test platform plate were important design considerations. A smooth, flat indentation tip with no fenestrations was used rather than actual grasper jaw geometry because a uniform circular flat tip ensures a constant and predictable contact profile between the material and the tip. This simplifies analysis as it avoids the difficulty of mapping applied pressure generated from a grasper jaw with fenestrations and a hinge mechanism. It also isolates strain effects on the tissue from the confounding effects of grasper geometry. Pressure would vary along the length of the jaw and depend on the mechanical advantage at the grasper linkage mechanism and would include areas of local stress at the peaks of the fenestrations. A large sample-to-tip dimension ratio also fulfills the half-space assumption used during analytical derivation for material testing. ${ }^{22}$

The full assembly schematics, .STL files and build of materials is available to use for free, via our Harvard Dataverse repository. ${ }^{23}$ Existing Python code was adapted and expanded to create custom software to control the actuator motion and hold its position once a predefined load threshold from the load cell was reached. ${ }^{24}$ This code is available to use freely on our Github repository. ${ }^{25}$

\section{Biospecimen tissue preparation and analysis}

Out of necessity, tissues included in this study were a convenience sample and were based on what operations were scheduled. All surgeons in the Division of General Surgery at St. Michael's Hospital were enrolled in the study and when they had a scheduled operation that included a surgical pathology sample (with wide enough margins of normal tissue), our research team would be present in the operating room and acquire the sample as soon as it was removed from the body (figure 1C). Samples were kept in a fresh state to preserve cellular integrity and mechanical properties. Once removed, samples were immediately taken to an adjacent histology suite where the two pathologists, CS and CR would assess the tissue so that sections for testing were taken from normal tissue not needed for pathological analysis. Six $1 \mathrm{~cm} \times 1 \mathrm{~cm}$ tissue squares were cut from each sample and each loaded onto the PrecisionCAT serosa side up for experimentation. Blue tissue marking dye (\#1003-5 Blue, Davidson Marking System, Minnesota, USA) was used to coat the indentation tip of the grasp plate before the experiment was performed so that when the tip made contact with the tissue, the area of compression would be evident when preparing and viewing slides. Once the experimental protocol was complete, the tested section was cut in half across the area of compression, fixed in formalin for normal histology processing and cut en-face to allow a full cross-sectional view across the area of compression. Tissues were fixed in $10 \%$ buffered formalin, processed and embedded in paraffin. Sections with a thickness of $4 \mu \mathrm{m}$ were cut and tissues were mounted to glass slides. Sections were stained with $\mathrm{H} \& \mathrm{E}$ for typical visualization of cell morphology and structure. Once slide preparation was complete, they were scanned at $20 \times$ using a brightfield digital pathology scanner. Slides were analyzed using digital slide viewing software (Aperio ImageScope, Leica Biosystems, Wetzlar, Germany). Serosal thickness was specifically targeted 


\begin{tabular}{|c|c|c|c|c|c|c|c|}
\hline $\mathbf{P}$ & Tissue type & $100 \mathrm{kPa}$ & $200 \mathrm{kPa}$ & $300 \mathrm{kPa}$ & $400 \mathrm{kPa}$ & $500 \mathrm{kPa}$ & $600 \mathrm{kPa}$ \\
\hline 1 & Small bowel & 0.477 & 0.073 & $<0.001$ & $<0.001$ & $<0.05$ & - \\
\hline 2 & Small bowel & 0.288 & 0.369 & - & 0.218 & - & $<0.001$ \\
\hline 3 & Small bowel & 0.358 & 0.495 & - & $<0.05$ & $<0.001$ & $<0.05$ \\
\hline 4 & Small bowel & 0.233 & 0.282 & 0.252 & $<0.05$ & $<0.001$ & $<0.001$ \\
\hline 5 & Small bowel & 0.369 & 0.342 & - & 0.057 & $<0.001$ & $<0.001$ \\
\hline 6 & Small bowel & 0.345 & 0.228 & $<0.05$ & $<0.05$ & $<0.05$ & $<0.05$ \\
\hline 7 & Small bowel & 0.474 & 0.455 & $<0.05$ & $<0.05$ & $<0.05$ & $<0.001$ \\
\hline 8 & Small bowel & 0.383 & 0.268 & $<0.001$ & $<0.001$ & $<0.001$ & $<0.001$ \\
\hline 9 & Small bowel & 0.432 & - & 0.449 & 0.475 & $<0.05$ & - \\
\hline 10 & Small bowel & - & 0.396 & 0.316 & - & $<0.001$ & $<0.001$ \\
\hline 11 & Colon & 0.409 & 0.052 & $<0.001$ & $<0.05$ & $<0.001$ & $<0.001$ \\
\hline 12 & Colon & 0.450 & 0.338 & 0.071 & $<0.05$ & $<0.001$ & $<0.05$ \\
\hline 13 & Colon & 0.246 & 0.492 & - & - & - & $<0.001$ \\
\hline 14 & Colon & 0.425 & 0.272 & $<0.05$ & - & $<0.05$ & $<0.001$ \\
\hline 15 & Colon & 0.316 & 0.400 & 0.065 & 0.187 & $<0.05$ & $<0.001$ \\
\hline 16 & Colon & - & 0.324 & 0.089 & - & $<0.05$ & $<0.001$ \\
\hline 17 & Colon & 0.482 & 0.330 & $<0.05$ & $<0.05$ & $<0.001$ & $<0.05$ \\
\hline 18 & Colon & 0.230 & 0.169 & 0.450 & - & 0.001 & $<0.001$ \\
\hline 19 & Colon & 0.091 & 0.301 & 0.442 & $<0.001$ & $<0.001$ & $<0.05$ \\
\hline 20 & Colon & 0.145 & $<0.05$ & $<0.05$ & $<0.001$ & $<0.05$ & $<0.001$ \\
\hline- & All tissue (group) & 0.450 & 0.410 & $<0.05$ & $<0.001$ & $<0.001$ & $<0.001$ \\
\hline- & $\begin{array}{l}\text { All sm. bowel } \\
\text { (group) }\end{array}$ & 0.398 & 0.444 & $<0.05$ & $<0.001$ & $<0.05$ & $<0.001$ \\
\hline- & All colon (group) & 0.479 & 0.236 & 0.095 & $<0.001$ & $<0.001$ & $<0.05$ \\
\hline
\end{tabular}

for quantification because it is serosal disruption that is hypothesized to be the basis of adhesion formation. ${ }^{26}$

\section{Tissue trauma score and serosa thickness calculations}

Due to the experimental protocol being ex-vivo, normal markers of cellular injury such as increased granulocyte recruitment (neutrophils and eosinophils) or apoptosis could not be used. Instead, areas of tissue injury were identified by quantifiable denudation of the normal layers of the intestine or from increased thinning and elongation of the cell with dense hyperchromatic nuclei. Two metrics of damage were quantified: an intestinal layer thickness calculation where the serosa (outermost) layer was measured in the area of compression $(\mathrm{C})$ and compared with a local control region that was not compressed as a per cent deformation and a histological scoring scale for tissue trauma (figure 2). The histological scoring scale was created by the two pathologists in this study as we were unable to find a suitable pathologist-validated scale endorsed in the literature. ${ }^{18}$ The criteria for the scale is outlined in table 1 and representative images shown in figure 2 .

Histological images were taken at $400 \times$ and all serosa layer thickness measurements were performed at the center of the experimental area of compression. Five measurements were taken of the thickness of the serosa layer in the area of compression and then averaged for a total value. Five measurements were also taken in an adjacent non-compressed area and averaged to serve as a local control. Per cent deformation (rather than an absolute delta in microns) was calculated as the per cent difference between the thicknesses of the compressed area to its local control of uncompressed tissue.

\section{Statistical analysis}

Statistical analysis and modeling was performed using NumPy, pandas and Scikit-learn using the Python programming language. ${ }^{27-29}$ All graphs were created with Matplotlib. ${ }^{30}$ A series of one-tailed t-tests were performed to compare sample serosal thickness measurements at compressed versus local control area in the histological slides. The tests were to investigate significant decrease in thickness due to compression of the tissue. To investigate the relationship between serosal thickness change and the pathologist's trauma score ratings, a correlation study was also completed.

A logistic regression model was trained to predict significant serosal thickness change as a classification task. A second logistic regression model was trained to predict a positive tissue trauma score. The intention 

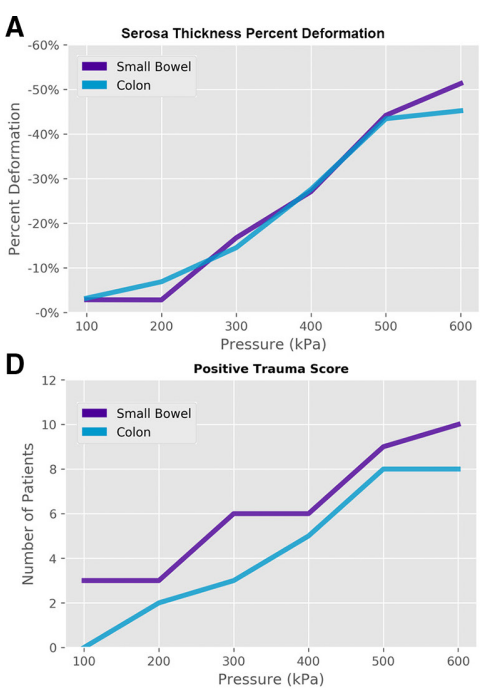
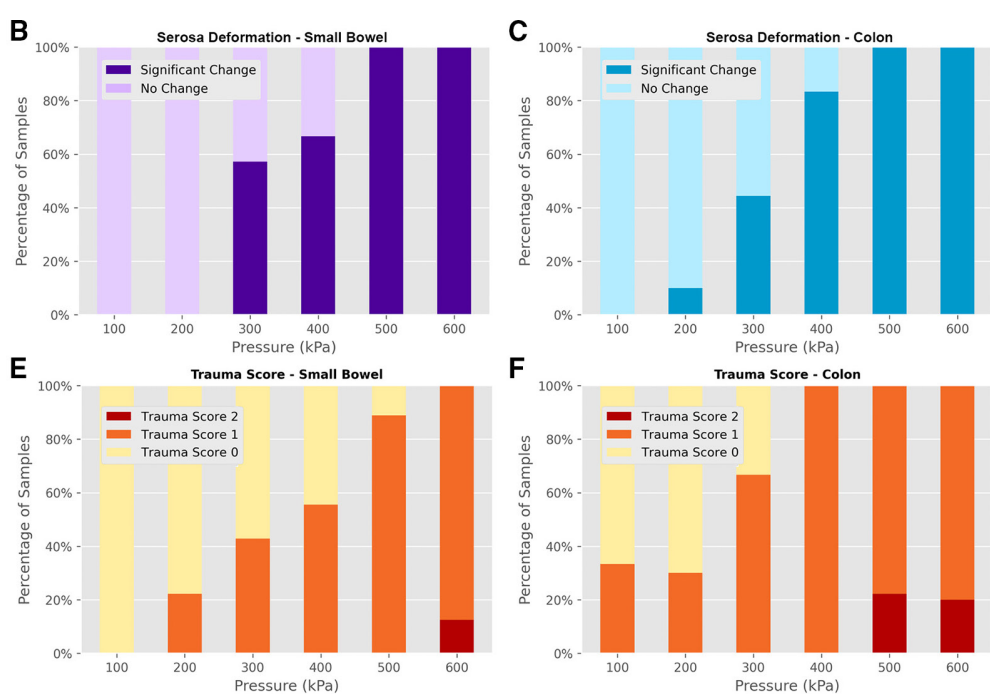

Figure 3 Serosal thickness change and Trauma Score. (A) A line graph of the serosal thickness per cent deformation by tissue type at each loading condition. $(B, C)$ The percentage of patients with significant $p$ values for serosal deformation by tissue type. (D) A line graph comparing positive trauma scores (either a Trauma Score of 1 or 2 indicating evidence of damage is present) of small bowel to colon tissues at each loading condition. (E, F) Stacked bar graphs of Trauma Score ratings at each loading condition by tissue type.

of these predictive models was evaluate how well input features that could be measured intraoperatively such as force, position of the grasper and time that the tissue was compressed and features that could be derived from those such as stress, strain and stiffness of the tissue, could be used to predict the likelihood of subsequent tissue trauma. This algorithm then could be incorporated into a "smart" laparoscopic tool that can, in real-time, alert a surgeon in the operating room about force use and subsequent pathological tissue response.

\section{RESULTS}

\section{Demographics and experimental cases}

Overall, 26 samples were procured but 6 tissue samples were unusable due to a variety of factors such as the pathologists being unavailable for immediate experimentation or the tissue was too inflamed. In total, we were able to complete the full experimental protocol on 20 patients' tissues, with 10 colon samples (1:1 male to women, mean age 65.2) and 10 small bowel (7:3 male to female, mean age 54.3) samples for a total of 120 experimental cases and 120 control cases.

\section{Tissue measurements}

\section{Slide creation and artifact rate}

As previously mentioned, the process of creating histological slides from tissue that has been subjected to a load force has an associated failure rate, which we have taken numerous steps from our previous study to optimise. Out of a possible 120 experimental tissue slides, a final useable total of 104 cases $(86.7 \%)$ were obtained.

\section{Average serosa layer thickness}

Average serosa thickness of both the small bowel $(0.284 \pm 0.225 \mathrm{~mm})$ and colon $(0.165 \pm 0.140 \mathrm{~mm})$ was calculated by averaging each tissue type's local control area measurements (excluding slides that had unsatisfactory histological slide creation). These values fall within range of those reported in the literature. ${ }^{31} 32$

\section{Serosa thickness deformation}

A series of paired t-tests were conducted between the five serosal control measurements and the five compression site measurements at each loading condition to determine significance (table 2 ), where $p$ values $\leq 0.05$ are indicated by bold text and missing values indicate that the slide was unable to be analyzed due to slide artifacts.

All 20 patients experienced a significant difference $(p<0.05)$ in serosal thickness postcompression at both 500 and $600 \mathrm{kPa}$ for both tissue types. The majority of patients at $400 \mathrm{kPa}(6 / 10$ for small bowel and $5 / 6$ for colon) also had significant differences precompression and post compression. This is similar to our pilot study where all patients had significant $\mathrm{p}$ values starting at $450 \mathrm{kPa}{ }^{18}$ This is also higher than the minimum pressure of $150 \mathrm{kPa}$ that Chandler et al s group found for damage to porcine colon, but their measurements were for the mucosal and muscle layers of the colon and did not focus on serosal change as they had this layer of tissue stripped. ${ }^{19}$ There were no significant changes at 100 and $200 \mathrm{kPa}$ (except for patient 20 for colon) and a mixed picture at $300 \mathrm{kPa}$ with $50 \%(8 / 16)$ of patients experiencing a significant difference between control and compressed tissues $(4 / 7$ for small bowel and 4/9 for colon). These data were visually displayed in figure 3B,C. When all small bowel patient data were combined together and analyzed by loading condition, the trend was similar to individual results. Significance is achieved at $300 \mathrm{kPa}$ and continues onwards from 400 to $600 \mathrm{kPa}$ with significant $\mathrm{p}$ values. This is similar to grouped colon patient data, where 
significance is achieved at $400 \mathrm{kPa}$ and continues onwards to $600 \mathrm{kPa}$ with significant $\mathrm{p}$ values. All patient data were then grouped together (both small bowel and colon) and analyzed by loading condition and this too followed a comparable pattern where significance is achieved at $300 \mathrm{kPa}$ and continues onwards from 400 to $600 \mathrm{kPa}$ with $p$ values of $<0.001$. Serosal thickness as a per cent deformation (to normalize measurements) was also calculated for each patient and plotted in figure 3A. Per cent deformation steadily increases as the experimental loading condition is increased and both tissue types follow similar patterns (figure 3B,C).

\section{Tissue trauma score}

Tissue trauma scores were assigned by both pathologists for each histological slide created based on the criteria outlined in table 1 (plotted in figure 3D). For small bowel (figure $3 \mathrm{E}$ ), at $600 \mathrm{kPa}$, all 8 patients experienced trauma to the serosa with $1 / 8$ patients graded as a Trauma Score of 2, and 7/8 patients being graded as a Trauma Score of 1 . At $500 \mathrm{kPa}, 8 / 9$ patients were graded with a Trauma Score of 1 with the remaining patients graded as no trauma. At $400 \mathrm{kPa}$, the picture is mixed as $5 / 9$ patients' tissues were graded with a Trauma Score of 1 and the remaining 4 patients displaying no trauma. At $300 \mathrm{kPa}, 3 / 7$ patients were graded with a Trauma Score of 1 while $4 / 7$ exhibited no trauma. At $200 \mathrm{kPa}$ only $2 / 9$ patients were graded with a Trauma Score of 1 and $7 / 9$ patients had no evidence of tissue trauma. Finally, at $100 \mathrm{kPa}$, no patient had a positive Trauma Score.

For colon (figure 3F), at $600 \mathrm{kPa}$, all 10 patients experienced trauma to the serosa with $2 / 10$ patients graded as a Trauma Score of 2, and 8/10 patients being graded as a Trauma Score of 1 . At $500 \mathrm{kPa}$, all 9 patients again had positive scores with $2 / 9$ patients graded with a Trauma Score of 2 with the remaining 7 patients graded with a Trauma Score of 1 . At $400 \mathrm{kPa}$, again all patients were rated as displaying evidence of trauma with all six patients receiving a score of 1 . At $300 \mathrm{kPa}$ the picture is mixed, with $6 / 9$ patients graded with a Trauma Score of 1 and the remaining three displaying no tissue damage. At $200 \mathrm{kPa}$, the majority $(7 / 10)$ of patients were rated with a score of 0 and $3 / 10$ patients graded with a score of 1 . Finally, at $100 \mathrm{kPa}$, again, the majority of patients $(6 / 9)$ were scored as displaying no trauma and $3 / 9$ being graded with a Trauma Score of 1 .

Out of 104 experimental samples, 83 samples (79.8\%) were classified in agreement between the two available metrics: Trauma Score and significant serosa thickness change. This means they were either both classified as having a positive Trauma Score and having significant serosa deformation or were both classified as having a Trauma Score of 0 and non-significant serosa deformation. Of the samples with disagreeing classification, $7 / 104$ $(6.7 \%)$ showed significant change in serosa thickness but were assigned a Trauma Score of 0 and 14/104 (13.5\%) were assigned a Trauma Score of 1 or greater but did not display evidence of significant serosa thickness change.

\section{Logistic regression model}

Two logistic regression models were trained in order to predict target metrics: a positive Trauma Score and significant serosa thickness change. Logistic regression was selected because it is an easily interpretable model and could be implemented in an intraoperative environment in a manual or automatic approach. The models were trained on a single feature (target stress, table 3), which was selected as it was the most predictive (it was the feature with the highest correlation). This feature was expected to be the most important to predict tissue trauma because it is directly derived from the maximum force.

The features that both models included were derived from the force and displacement measurements obtained during each experimental condition. First, the force measurements were transformed to stress on the tissue based on the contact surface area of the

\section{Table 3 Logistic regression model features}

\begin{tabular}{ll}
\hline Feature & Definition \\
\hline Target stress $(\sigma)$ & $\begin{array}{l}\text { The peak compressive stress in the tissue measured in MPa induced by the pin plate's compression } \\
\text { of that tissue at the target load. }\end{array}$ \\
\hline Target duration & $\begin{array}{l}\text { The length of time in seconds in which the indentation pin holds its end position in contact with the } \\
\text { tissue, measured in seconds. }\end{array}$ \\
\hline Compression duration & $\begin{array}{l}\text { The length of time in seconds in which the tissue is being actively compressed before the target } \\
\text { stress is reached. }\end{array}$ \\
Contact stiffness & $\begin{array}{l}\text { The lowest measured stiffness (MPa) which occurs at initial contact, where stiffness is defined as } \\
\text { stress/strain. }\end{array}$ \\
\hline Target stiffness & $\begin{array}{l}\text { The maximum measured stiffness (MPa) which occurs when the target stress is achieved, where } \\
\text { stiffness is defined as stress/strain. }\end{array}$ \\
\hline Relaxation stress & $\begin{array}{l}\text { The decrease in stress on the tissue (in MPa) as the indentation pin holds its position and tissue } \\
\text { components such as fluids shift causing a dynamic reduction in stiffness. }\end{array}$ \\
Initial thickness & $\begin{array}{l}\text { The initial contact thickness of the tissue in mm. } \\
\text { The peak compressive strain (per cent deformation) that the indentation pin induces in the tissue } \\
\text { relative to its initial thickness. }\end{array}$ \\
\hline
\end{tabular}


indentation pin. The displacement measurements, measured with a resolution of $1 \mu \mathrm{m}$, were converted into strain of the tissue, taking into account the initial tissue thickness, as determined by the tool coming into contact with the tissue. Both of these measurements, in addition to time stamps, could be measured in a sensorized grasper tool.

From these data, further features were derived, which is shown in table 3. Additionally, patient age, gender and tissue type were included as features as well.

\section{Serosa deformation model}

The first model to predict significant serosa deformation uses all the features derived from the force, position and time measurement data. It is able to achieve a sensitivity of $100 \%$ and a specificity of $84.6 \%$ on a test set of data (20\% of total cases). The receiver operating characteristic curve shows the performance of the classification model at all thresholds and can be used to assess its performance as compared with random guessing. The area under the curve (AUC) is $92 \%$.

The model was retrained using only target stress as an input feature. The advantage of this restricted approach is that it only needs intraoperative force measurements which potentially can simplify what sensors are needed in a sensorized grasper. The performance of the model deteriorated relative to the full featured model, with sensitivity dropping to $75 \%$ and specificity remaining the same at $84.6 \%$. The AUC dropped to $80 \%$.

\section{Trauma Score model}

The first model to predict positive Trauma Score using all the features, is able to achieve a sensitivity of $76.9 \%$ and a specificity of $100 \%$ on a test set of data $(20 \%$ of total cases). The AUC is $88 \%$.

The model was retrained using only target stress as an input feature. The performance of the model deteriorated relative to the full featured model, with sensitivity dropping to $61.5 \%$ and specificity remaining the same at $100 \%$. The AUC dropped to $81 \%$.

\section{Model analysis}

The first metric of significant serosa deformation with all features has a high sensitivity and thus will identify all cases of trauma successfully but may have some false positives. The second metric of positive Trauma Score has a high specificity and thus will identify patients who do not have significant trauma but may have some false negatives. There may be value in applying both models as conservative prediction is preferred.

Both models showed a deterioration when features were restricted to target stress only. There may be advantages in implementation however in only measuring intraoperative forces with sensorized tools, so this degradation in performance is presented as a comparison. The suitability of these restricted models would need to be evaluated in a clinical setting.

\section{DISCUSSION}

This paper aimed to build on our previous work with a more sophisticated compressive device that had the additional capabilities of force, time and position logging. We were also able to optimize both our histology slide preparation protocol and patient recruitment to run a more comprehensive study. Despite the importance of elucidating the relationship between compressive force and tissue trauma, only a few studies have been performed to date. As far as we know, we are the first group to publish a paper based on compressive data of human gastrointestinal tissues via a histological analysis with such a large amount of patient tissue data. The histological analysis was central to this study as it allowed us to objectively identify at which loading conditions mechanical trauma occurred.

Heijnsdijk's work with porcine and human bowel tissues is that the inter-individual variability in perforation forces is large and that bowel strength could differ by a factor of two between patients. ${ }^{17}$ Taking this into account we must be extremely careful and conservative when defining "safe" force limits for intestinal tissue because a force that could be safely applied to one patient may cause a perforation in another patient.

Using our logistic regression analysis, our data points in the direction of establishing a maximum force cutoff starting at $329 \mathrm{kPa}$ on average for gastrointestinal tissues using a 50\% threshold, however, very large safety margins should be considered and used. This is similar to the results of our pilot study which suggested a damage threshold of $350 \mathrm{kPa}$ for colon tissues. ${ }^{18}$ These results need to be viewed in context of the limitations of an ex-vivo, in-vitro study, with the chief caveat revolving around the inability to see how these forces affect tissue in-vivo. How much serosal damage can the body repair and at what point does the repair mechanism become dysfunctional and leads to adhesion formation or necrosis needs to be further elucidated in a longitudinal, in-vivo study that can quantify objective markers of inflammation and cellular death.

In context of the current existing literature, Heijnsdijk's study saw perforation of the human small bowel at $10.3 \pm 2.9 \mathrm{~N}$ of force, but that was using a sharp pinch device with a smaller diameter than our pin plate (thus generating higher pressure loads), with weights that were manually loaded and moved by the experimenter. Christensen et als team showed trauma occurring at $150 \mathrm{kPa}$ but that was in a porcine model, and porcine tissues have a lower ultimate tensile strength and elastic modulus than human tissues do. ${ }^{16}$ What our study makes clear and confirms from these two previous studies is that tissue damage definitively correlates to how much force is exerted on that tissue in compression. The two pathologists who were blinded to the loading condition of the tissue were both independently able to quantify increasing damage amounts to increasing pressure; this relationship was true regardless of the tissue type. Our study is a foundational study that other groups may 
build on using our standardized methodology. We have released the entire build of materials, schematics, threedimensional-printing files and Python code necessary to replicate this study and invite research groups that would like to reproduce our study to do so, as multisite validation with a much larger $n$ number than we could produce is necessary to authenticate our results.

A limitation of this study was the fact that it is an ex-vivo study so that other histological findings of trauma such as microvascular changes to the endothelial cells or margination of neutrophils and the development of microthrombi could not be evaluated. These markers of damage require that the tissue be perfused for at least a few minutes to a full hour after compression. This level of analysis could be investigated in a future study where tissue resected as part of the normal surgical workflow that had known clamp times/compression stress could be evaluated post-removal. We also limited our analysis to laparoscopic graspers but the issue of tissue trauma from excessive force also applies to a variety of other common laparoscopic tools such as tenaculum, retractors or articulating forceps. Future studies could also perform our protocol on other delicate tissue types such as fallopian tubes or ureters.

Our paper adds strong evidence for the practical use of this information, namely, that if an upper limit of atraumatic force can be reliably established in humans, this force cut-off should be used intraoperatively via laparoscopic graspers "smart" tools. The two logistic regression equations created could be used in a tool intraoperatively, provided that the features used could be measured in real-time. This would require a minimum of two sensors: one for force and one for grasper jaw position, that would measure these in-vitro. Sensorized laparoscopic graspers can provide real-time force information to an operating surgeon, who can then limit themselves to this "safe zone." One of the biggest hurdles to surmount with the adoption and practical implementation of sensorized tools however is the need to sterilize these tools at high temperatures or via harsh chemicals which may corrode the sensors and produce invalid measurements.

Of course, force limits would be tissue-type specific as a tissue's ability to handle pressure is a function of its underlying cellular structure. However, the outcomes of this study are a promising step forward in the field of tissue trauma prediction and prevention in surgery.

Acknowledgements We would like to first thank all of the patients who participated in our study and graciously allowed us the use of their tissues for the advancement of science. Second, the Hospital for Sick Children's Perioperative Services Grant kindly funded the creation of the PrecisionCAT in collaboration with the Centre for Image-Guided Innovation and Therapeutic Intervention (CIGITI). We also extend our gratitude to the general surgeons from St. Michael's Hospital in Toronto who allowed us into their operating rooms, Shehryar Saharan for his assistance with creating the figures in our paper and Dr Shuning "Steve" Bian for allowing us to adapt his Python code. We are also in debt to Drs Peter Culmer and James Chandler from the School of Mechanical Engineering at the University of Leeds who created the original crush apparatus that inspired our design. We would also like to thank Drs Anne Agur, Ted Gerstle, Joel Krivy and Hani Naguib for their wisdom, brainstorming and feedback, which this paper would not be published without. Finally, we would like to thank our long-time collaborator Granitus MKM for her always useful input, proofreading and commentary on the manuscript. Portions of this manuscript have been previously published as part of AFK's PhD dissertation.

Contributors AFK contributed to the research study design, creation and fabrication of the force device, data acquisition, data analysis and manuscript preparation. MKM contributed to the research study design, creation and fabrication of the force device, software coding, data analysis and manuscript preparation. CS contributed to the research study design, data acquisition, data analysis and manuscript preparation. CR contributed to the research study design, data acquisition, data analysis and manuscript preparation. JD contributed to the research study design and supervision. TG contributed to the research study design and supervision.

Funding The Hospital for Sick Children's Perioperative Services Grant Project $\# 121$.

Competing interests None declared.

Patient consent for publication Not required.

Ethics approval A study to test the device was approved by the Research Ethics Office of St. Michael's Hospital (REB \#15-299) in Toronto, Ontario.

Provenance and peer review Not commissioned; externally peer reviewed.

Data availability statement Data are available in a public, open access repository. All files and schematics used to create the PrecisionCAT is freely available on Harvard's Dataverse Repository at the following URL: https://dataverse.harvard. edu/dataset.xhtml?persistentld=doi:10.7910/DVN/QOWOEZ. Code Availability: All custom Python code created and used in the preparation of this manuscript is freely available on Github. The PrecisionCAT data is available, freely to the public at the following URL: https://github.com/crushdevice.

Open access This is an open access article distributed in accordance with the Creative Commons Attribution Non Commercial (CC BY-NC 4.0) license, which permits others to distribute, remix, adapt, build upon this work non-commercially, and license their derivative works on different terms, provided the original work is properly cited, appropriate credit is given, any changes made indicated, and the use is non-commercial. See: http://creativecommons.org/licenses/by-nc/4.0/.

\section{ORCID iD}

Amanda Farah Khan http://orcid.org/0000-0002-8834-4435

\section{REFERENCES}

1 Moore M. Minimally invasive procedures in hysterectomy, appendectomy, cholecystectomy \& colectomy. Ethicon EndoSurgery - Value Dossier 2009.

2 Bishoff JT, Allaf ME, Kirkels W, et al. Laparoscopic bowel injury: incidence and clinical presentation. J Urol 1999;161:887-90.

3 Barrie J, Russell L, Hood AJ, et al. An in vivo analysis of safe laparoscopic grasping thresholds for colorectal surgery. Surg Endosc 2018;32:4244-50.

4 van der Voort M, Heijnsdijk EAM, Gouma DJ. Bowel injury as a complication of laparoscopy. Br J Surg 2004;91:1253-8.

5 Tang B, Hanna GB, Joice P, et al. Identification and categorization of technical errors by observational clinical human reliability assessment (OCHRA) during laparoscopic cholecystectomy. Arch Surg 2004;139:1215-20.

6 Berwick DM. Errors today and errors tomorrow. N Engl J Med 2003;348:2570-2.

7 Leape LL, Brennan TA, Laird N, et al. The nature of adverse events in hospitalized patients. Results of the Harvard medical practice study II. N Engl J Med 1991;324:377-84.

8 Baker GR, Norton PG, Flintoft V, et al. The Canadian adverse events study: the incidence of adverse events among hospital patients in Canada. CMAJ 2004;170:1678-86.

9 Gupta V, Reddy NP, Batur P. Forces in surgical tools: comparison between laparoscopic and surgical forceps. Proceedings of the 18th Annual International Conference of the IEEE Engineering in Medicine and Biology Society, 1996:223-4.

10 Marucci DD, Shakeshaft AJ, Cartmill JA, et al. Grasper trauma during laparoscopic cholecystectomy. Aust N Z J Surg 2000;70:578-81.

11 Carniel EL, Gramigna V, Fontanella CG, et al. Characterization of the anisotropic mechanical behaviour of colonic tissues: experimental activity and constitutive formulation. Exp Physiol 2014;99:759-71.

12 Ishijima A, Koseki Y, Ando T, et al. Preclinical methods to assess collateral mechanical damage to tissues caused by surgical procedures and devices. JJSCAS 2016;18:19-32. 
13 Marucci DD, Cartmill JA, Walsh WR, et al. Patterns of failure at the instrument-tissue interface. J Surg Res 2000;93:16-20.

14 Cheng L, Hannaford B. Finite element analysis for evaluating liver tissue damage due to mechanical compression. J Biomech 2015;48:948-55

15 De S, Rosen J, Dagan A, et al. Assessment of tissue damage due to mechanical stresses. Int J Rob Res 2007:26:1159-71.

16 Christensen MB, Oberg K, Wolchok JC. Tensile properties of the rectal and sigmoid colon: a comparative analysis of human and porcine tissue. Springerplus 2015;4, :142.

17 Heijnsdijk EAM, van der Voort M, de Visser $\mathrm{H}$, et al. Inter- and Intraindividual variabilities of perforation forces of human and pig bowel tissue. Surg Endosc 2003;17:1923-6.

18 Khan AF, Macdonald MK, Streutker C, et al. Defining the relationship between compressive stress and tissue trauma during laparoscopic surgery using human large intestine. IEEE J Trans/ Eng Health Med 2019;7:1-8

19 Chandler JH, Mushtaq F, Moxley-Wyles B, et al. Real-Time assessment of mechanical tissue trauma in surgery. IEEE Trans Biomed Eng 2017;64:2384-93.

20 Barrie J, Jayne DG, Neville A, et al. Real-Time measurement of the Tool-Tissue interaction in minimally invasive abdominal surgery: the first step to developing the next generation of smart laparoscopic instruments. Surg Innov 2016;23:463-8.

21 Brown JD, Rosen J, Chang L, et al. Quantifying surgeon grasping mechanics in laparoscopy using the blue dragon system. Stud Health Technol Inform 2004;98:34-6.

22 Wang J, Yu Q-Y, Li W, et al. Influence of clamping stress and duration on the trauma of liver tissue during surgery operation. Clin Biomech 2017;43:58-66.
23 Wang X, Schoen JA, Rentschler ME. A quantitative comparison of soft tissue compressive viscoelastic model accuracy. J Mech Behav Biomed Mater 2013;20:126-36.

24 Harvard DataverseKhan A. PrecisionCAT Solidworks assembly files and build of materials, 2018. Available: https://dataverse.harvard. edu/dataset.xhtml?persistentld=10.7910/DVN/QOWOEZ [Accessed 1 Oct 2018].

25 Bian S. Smac-lac-1 code, 2016. Available: https://github.com/ freespace/smac-lac-1/blob/master/lac1.py

26 Khan A, MacDonald M, GitHub PrecisionCAT Repository. PrecisionCAT GitHub Repository, 2019. Available: https://github. com/crushdevice

27 Brochhausen C, Schmitt VH, Planck CNE, et al. Current strategies and future perspectives for intraperitoneal adhesion prevention. $J$ Gastrointest Surg 2012;16:1256-74.

28 van der Walt S, Colbert SC, Varoquaux G. The NumPy array: a structure for efficient numerical computation. Comput Sci Eng 2011;13:22-30.

29 SCRIBD, Database Index. Pandas: a foundational python library for data analysis and statistics. Available: https://www.scribd.com/ document/71048089/pandas-a-Foundational-Python-Library-forData-Analysis-and-Statistics [Accessed 25 Mar 2020].

30 Pedregosa Fabianpedregosa F. Scikit-learn: machine learning in python, 2011. Available: http://scikit-learn.sourceforge.net [Accessed 05 Jan 2019].

31 Hunter JD. Matplotlib: a 2D graphics environment. Comput Sci Eng 2007;9:90-5.

32 Cronin CG, Delappe E, Lohan DG, et al. Normal small bowel wall characteristics on MR enterography. Eur J Radiol 2010;75:207-11 